\title{
A fuzzy logic approach to image analysis of HREM micrographs of III-V compounds
}

\author{
R. Hillebrand*, P. Werner, H. Hofmeister, U. Gösele \\ Max-Planck-Institut für Mikrostrukturphysik, Weinberg 2, D-06120 Halle Saale, Germany
}

Received 15 April 1996; accepted 7 October 1996

\begin{abstract}
High resolution electron microscopy can within certain limits provide quantitative information on morphology and composition of crystalline materials. In layered structures of III-V semiconductor compounds changes resulting from interdiffusion phenomena across interfaces may result in image contrast changes because of composition sensitive electron scattering. A new approach for extracting the corresponding information from cross-section images of such interfaces is introduced. Local variations of similarity, a measure of comparability with respect to previously defined templates of known material composition on both sides of the interface, are recorded by image analysis. On the basis of the well-worked out fuzzy set theory variations of the underlying chemical composition are evaluated by applying fuzzy logic criteria of inference to image regions of about $1 \mathrm{~nm} \times 1 \mathrm{~nm}$ in size.

With comparatively little computational effort this new approach can be used to localize regions of significant composition changes, i.e. edge detection, and to determine the composition across the interface region, i.e. chemical mapping, respectively. The method is introduced and its reliability demonstrated by means of simulated contrast patterns of $\mathrm{GaAs} / \mathrm{GaAs}_{1-x} \mathrm{P}_{x}$ as well as of experimental images of $\mathrm{AlAs} / \mathrm{Al}_{x} \mathrm{Ga}_{1-x} \mathrm{As}$.
\end{abstract}

\section{Introduction}

Advanced technologies of semiconductor crystal growth such as molecular beam epitaxy (MBE) allow the production of layered structures of III-V compounds like GaAs and associated heterostructures with nearly atomically smooth interfaces. Essential physical properties of opto-electronic devices directly depend upon the quality of these

* Corresponding author. interfaces, which is also governed by interdiffusion processes. For this reason, quantum well and superlattice structures of compound semiconductors are increasingly studied. Besides spectroscopic and analytical methods, high resolution electron microscopy (HREM) provides highly resolved lateral information on this subject.

For studying the quality of interfaces as well as the interdiffusion in layered III-V compounds structural and compositional information can be obtained from HREM micrographs by quantitative image analysis. Already established techniques like chemical mapping developed by Ourmazd et al. [1] 
and its more general extension QUANTITEM by Schwander et al. [2] are based on vector pattern recognition methods. Substantial contributions to chemical mapping of III - V compounds have also been given by De Jong and Van Dyck [3], Thoma and Cerva [4] and Walther et al. [5]. In contrast to these in the present paper a fuzzy logic approach is introduced, initiated by a paper of Tyan and Wang [6], which also attains near-atomic resolution, given by the size of crystal sub-cells of the material imaged.

On the basis of two image templates of material with known composition distant from the interface, similarity values $S$ of small image areas (cells) are determined by a difference measure. The similarity of neighbouring cells within appropriately defined masks about $1 \mathrm{~nm} \times 1 \mathrm{~nm}$ in size is evaluated in order to draw conclusions about the variations of the composition $C$, especially at distinct compositional boundaries. The fuzzy set theory $[7,8]$ allows fuzzy logic $(F L)$ inference criteria to be adapted to the correlation between local similarity and composition. The inference criteria are formulated in fuzzy rules and applied to the physically based types of membership functions, which involve the similarity behaviour related to the templates.

The fuzzy logic approach described is the application of a well-worked out mathematical theory. The kind of membership functions as well as the fuzzy rules have to reflect the physical problem to be solved. For the triangular membership functions derived, the FL inference meets the physical requirements, i.e. the "conservation of matter (total amount of atoms in the sublattices is constant)' and the 'monotonicity of the chemical gradient'. In FL, there is no need to formulate, approximate and solve any analytical apparatus for this dynamical and non-linear problem. The characteristics of deducing the composition from similarity variations are derived from results of contrast simulations. The approach makes use of the one-to-one relation of similarity and chemical composition under appropriate experimental conditions. This assumption is critically discussed for different materials and crystallographic orientations, with two topics being treated in detail in the present paper:

(i) the localization of image regions with significant changes in composition (edge detection), (ii) the chemical mapping of composition across the interface region.

In Section 2 the techniques of composition mapping and edge detection will be introduced and discussed on the basis of simulated HREM images. After a description of material aspects and data acquisition techniques, the development of the FL formalism will be described and graphically illustrated. In Section 3 experimental micrographs of III-V materials, exhibiting modifications in one sublattice owing to growth (layer structures) or physical treatment (interdiffusion), will quantitatively be analysed by the fuzzy logic tools.

\section{Method}

\subsection{HREM and materials aspects}

The simulation of HREM images according to structure models with realistic properties is an appropriate means of developing and testing algorithms of image analysis. Useful experimental conditions such as, e.g., the optimum defocus can be pre-determined from calculated contrast tableaus. In the following, HREM image simulations of GaAs with a variable content of $\mathrm{P}$ in the group $V$ sublattice are used. We are specifically interested in the group $\mathrm{V}$ sublattice, since in contrast to the reasonably good knowledge of diffusion processes in the group III sublattice of GaAs, see, e.g., Ref. [9], very little is known about diffusion processes and point defects in the group $\mathrm{V}$ sublattice.

The scattering behaviour of a statistical mixture of two different elements in one sublattice (e.g., Al, $\mathrm{Ga}$, or for the case of interest As, P) can be approximated by the so-called VCA-interpolation (see also [10]). If, for instance, the atomic scattering factors are interpolated according to changes of the As/P ratio, the intensity of the $\left\{\begin{array}{lll}2 & 0 & 0\end{array}\right\}$ reflections varies strongly with the statistical increase of the $P$ content. The strength of the $\left\{\begin{array}{lll}2 & 0 & 0\end{array}\right\}$ reflections may be interpreted as the "degree of polarity" of the $\mathrm{GaAs}_{1-x} \mathrm{P}_{x}$ crystals. For image contrast simulations, commercial computer programs are available, e.g., EMS [11], with the "multi-slice" algorithm implemented for the dynamical diffraction within the crystal and the general non-linear 
image formation theory considered. The physical background of chemical mapping techniques (cf. [12]) is based on the variation of electron scattering according to the composition of the III-V crystals. Apart from the chemical composition of the crystals, specimen thickness and imaging parameters strongly influence the image patterns.

Fig. 1a shows HREM patterns simulated from $\left\langle\begin{array}{lll}0 & 0 & 1\end{array}\right\rangle$-oriented $\mathrm{GaAs}_{1-x} \mathrm{P}_{x}$ crystals for an accelerating voltage of $400 \mathrm{kV}$ and $\mathrm{C}_{\mathrm{s}}=1 \mathrm{~mm}$ by means of the EMS package [11]. This tableau is made up of crystallographic cells, each represented by $32 \times 32$ pixels, with the crystal thickness linearly increasing from bottom $(5 \mathrm{~nm})$ to top $(17 \mathrm{~nm})$. To guarantee highly selective imaging conditions an
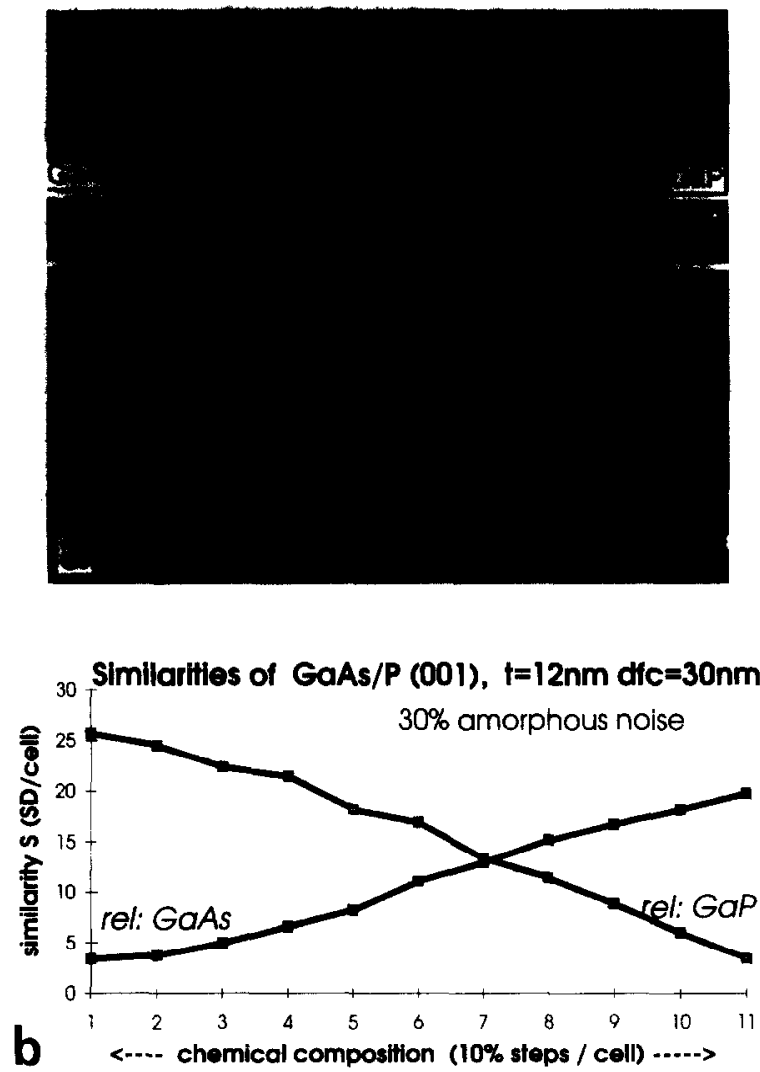

Fig. 1. (a) HREM contrast tableau of $\mathrm{GaAs}_{1-x} \mathrm{P}_{x}\langle 001\rangle$ with varying As/P-composition (horizontal) and $30 \%$ additive amorphous noise. Accelerating voltage $U=400 \mathrm{kV}, \mathrm{C}_{s}=1 \mathrm{~mm}$ (insets: templates), $t=12 \mathrm{~nm}$. (b) SD-profiles of similarity, related to the GaAs- and GaP-templates. underfocus of $30 \mathrm{~nm}$ is used here as the optimum value which was determined by preliminary studies with the defoci varying from $-25 \mathrm{~nm}$ (overfocus) to $75 \mathrm{~nm}$ (underfocus). In the horizontal direction, the As/P ratio is varied in steps of $10 \%$. To approach realistic conditions, additive amorphous noise is included in the image simulations, which amounts to $30 \%$ of the total contrast with the Fourier characteristics of the corresponding contrast transfer function.

A systematic comparison of the resulting contrast patterns shows that their relative similarity correlates with variations of the chemical composition, even when amorphous noise is present. From a number of similarity measures tested and published $[13,14]$, the standard deviation SD of the difference pattern of two compared images proved to be nearest to linearity. The measure of similarity $S$ of two images $I_{u}$ and $I_{v}$ having a cell size of $m \times n$ pixels reads:

$S=\sigma^{m, n}\left\{I_{u}^{m, n}-I_{v}^{m, n}\right\}$

$\sigma-$ standard deviation (SD).

Using Eq. (1) with the two internal templates of GaAs and GaP shown as insets in Fig. la yields two series of similarity values along the marked row. The corresponding profiles plotted in Fig. 1b represent the similarity/composition characteristics in the pre-determined experimental parameter window.

Fig. 2a presents the zoomed GaAs and GaP templates to outline clearly the problem of image cell comparison. As shown in Fig. $2 \mathrm{~b}$ and discussed in [15] the characteristics are sensitive to any noise in the image and to grid misalignment with respect to the template. The solid line represents the case of perfect alignment free of noise; the curve is less steep for $30 \%$ amorphous noise and does not attain the value $S=0$ (identity). A grid shift of two pixels essentially disturbs this characteristic relative to the slope for perfect alignment. For crystal cells of $32 \times 32$ pixels in size an axial template shift by 16 pixels corresponds to the translation vector of periodicity as the self-similarity profile of Fig. $2 c$ shows. For direct comparison of the GaAs and $\mathrm{GaP}$ templates, the difference measure of similarity 


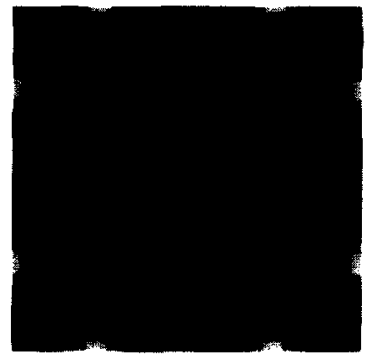

GaAs

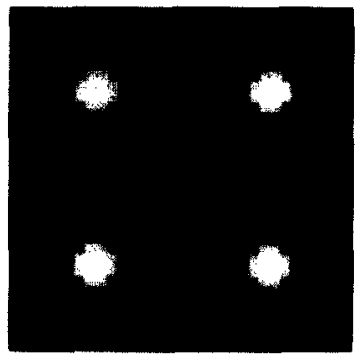

GaP

8

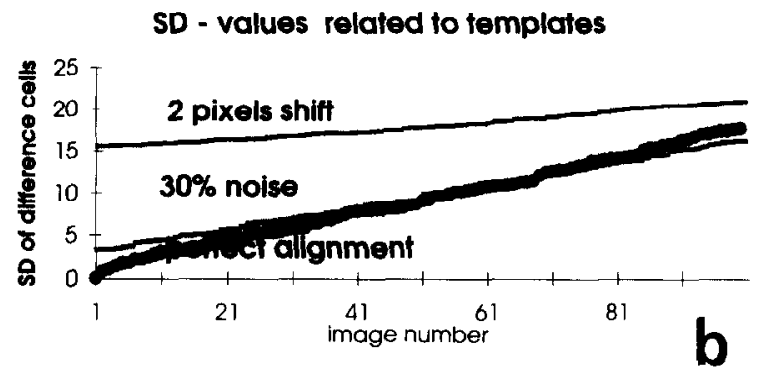

GaAs/P(001) - similarity and template shift

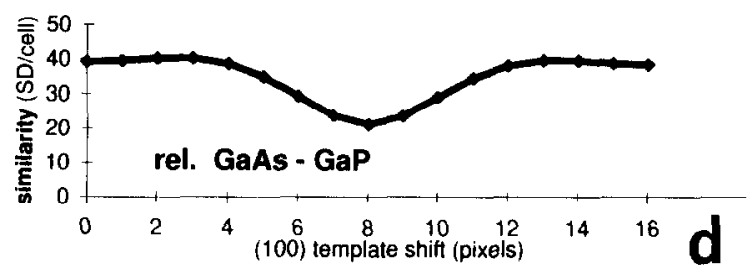

Fig. 2. (a) GaAs and GaP templates $(t \approx 12 \mathrm{~nm}, \Delta=30 \mathrm{~nm})$ and plots of similarity profiles: (b) ideal, $30 \%$ additive amorphous noise, and $12 \%$ lateral grid shift, (c) lateral template shift $\mathrm{GaAs}-\mathrm{GaAs}$, (d) lateral template shift $\mathrm{GaAs}-\mathrm{GaP}$.

cannot drop below a certain level as plotted in Fig. 2d. Even for arbitrary grid-mismatches there will be no pattern fit. The effects of noise, misalignment and geometrical specimen imperfections on chemical mapping are discussed in more detail elsewhere $[12,16]$.

For reasons of compatibility with experimentally recorded images, a specimen model of P-poor GaAs/P $(P \leqslant 50 \%)$ is established based on a stepwise chemical conversion from GaAs to $\mathrm{GaAs}_{0.5} \mathrm{P}_{0.5}$. The simulated image shown in Fig. 3 is calculated for $\langle 001\rangle$ crystal orientation and appropriate imaging conditions (crystal thickness $t \approx 11 \mathrm{~nm}$, defocus $\Delta \approx 30 \mathrm{~nm}$ ). Four interface structures are observed having different barrier gradients of chemical composition ranging from a slight linear variation of the As/P ratio (region I) to an abrupt As/P step (region IV). A certain roughness of the interfaces is modelled by considering composition changes in the diagonal image direction. This model with amorphous noise added proved to be an appropriate test object for the development of the FL approach.

For image analysis, all cells of the HREM image simulated are compared with the respective cells of two internal reference templates, viz. pure GaAs and $\mathrm{GaAs}_{0.5} \mathrm{P}_{0.5}$, yielding two matrices of similarity. The 3D relief of Fig. 4 displays the similarity values normalized for the barrier-type composition model of Fig. 3 (see also Ref. [17]). On the basis of these similarity matrices, the fuzzy logic classification and analysis will be discussed in detail in the next sections.

\subsection{Image analysis}

As Figs. 1 and 2 demonstrate the similarity values of $\mathrm{GaAsP}\langle 001\rangle$ show a quasi-linear correlation with the variation of the chemical composition. The same behaviour has been found for the system AlGaAs. Considering the simulated HREM image of Fig. 3 the similarity $S$ of any image cell $I_{i, j}$ to the reference templates of $\mathrm{GaAs}$, marked by $A$, and to $\mathrm{GaAs}_{0.5} \mathrm{P}_{0.5}$, marked by $\mathrm{B}$, is defined by

$$
\begin{aligned}
& \sigma\left\{I_{i, j}^{m, n}-A^{m, n}\right\}={ }_{\text {def }} S_{i, j-} A, \quad i=1, \ldots, N \_ \text {row }, \\
& j=1, \ldots, N \_ \text {col, } \\
& \sigma\left\{I_{i, j}^{m, n}-B^{m, n}\right\}={ }_{\text {def }} S_{i, j-} B .
\end{aligned}
$$




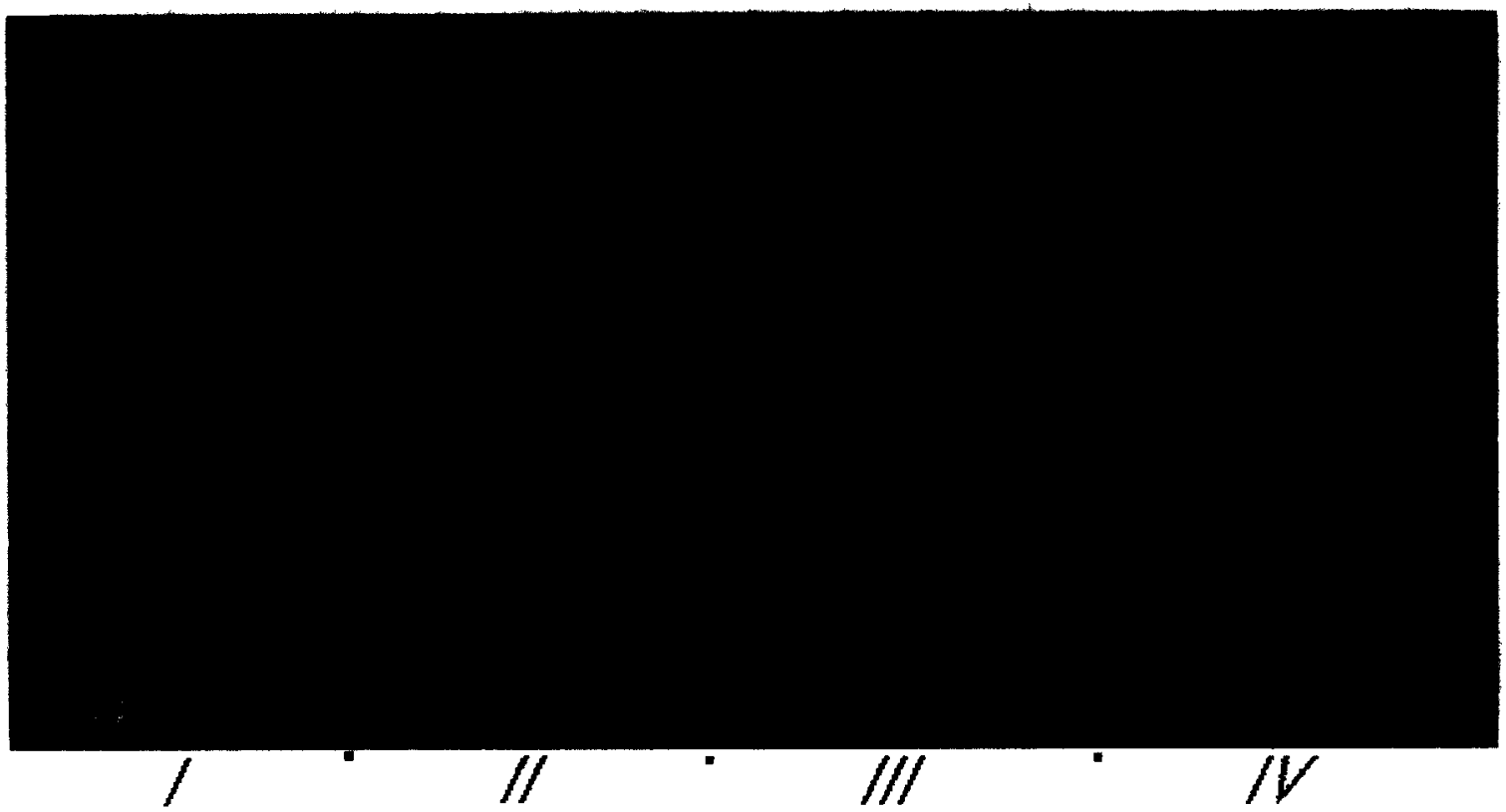

Fig. 3. Simulated HREM image $(16 \times 32$ cells $)$ of $\mathrm{GaAs}_{1-{ }_{x}} \mathrm{P}_{x}(001)$ with varying $\mathrm{As} / \mathrm{P}$-composition $(0 \leqslant x \leqslant 0.5)$ and $30 \%$ amorphous noise according to four models of composition change [I-III - different linear slopes, IV - As/P step]. Voltage $U=400 \mathrm{kV}$, crystal thickness $t=11 \mathrm{~nm}$, spherical aberration $C_{\mathrm{s}}=1 \mathrm{~mm}$.

\section{SImllarty reliets of 4 different GaAs/P barrier models}

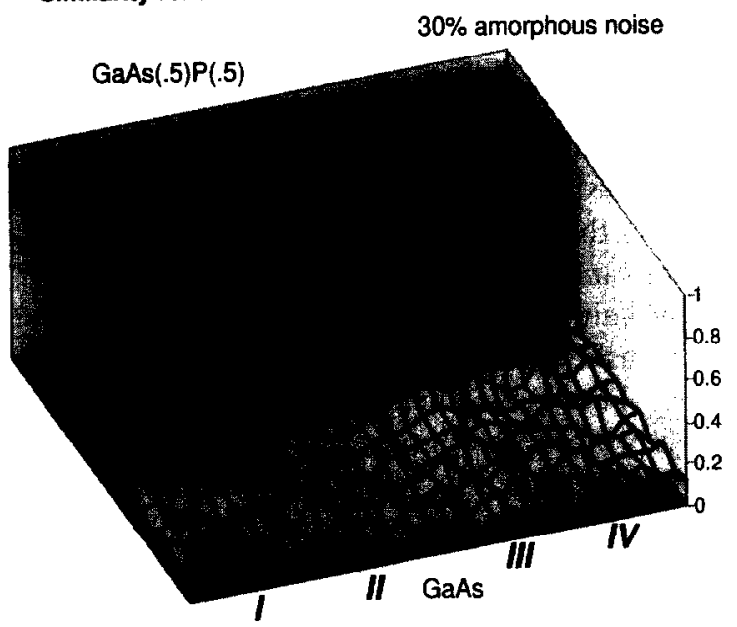

Fig. 4. Relief of similarities ( $\sigma /$ cell, related to the templates) of Fig. 3. i.e. the four $\mathrm{GaAs}_{0.5} \mathrm{P}_{0.5} / \mathrm{GaAs}$ barriers modelled $(30 \%$ noise).

The $N_{-}$row $\times N_{-}$col-sized matrices of similarity are calculated cell by cell $(32 \times 32$ pixels $)$ and normalized over the closed interval of $S \in[0,1]$. As the plots of Fig. $1 \mathrm{~b}$ indicate, the distributions of sim- ilarity values given by Eq. (2) are almost complementary. The deviations are mainly due to the non-linear characteristics of the electron-optical imaging process and the amorphous noise. Statistical analysis of the images revealed a maximum error of $4 \%$ for the values of similarity. Fig. 4 shows the similarity relief of the structure model based on four different composition gradients. It is obvious that the values of similarity are noisy. This variable model of an interface is used to test the $\mathrm{FL}$ rules to be established.

To illustrate the basics of the FL approach and to derive the necessary set of FL rules requires the analysis of histograms of cell similarities. The histograms are calculated by classifying the values of $S \in[0,1]$ into a variable number of equidistant intervals of similarity. Fig. 5 is obtained by counting each cell of the simulated micrograph (cf. Fig. 3) into one interval of similarity values. In the example given the values of $S$ are related to template $\Lambda$, i.e. GaAs displays close similarity, whereas the B-type image areas $\left(\mathrm{GaAs}_{0.5} \mathrm{P}_{0.5}\right)$ yield higher values for $S$. The histogram of these values clearly shows two peaks corresponding to two modes with 


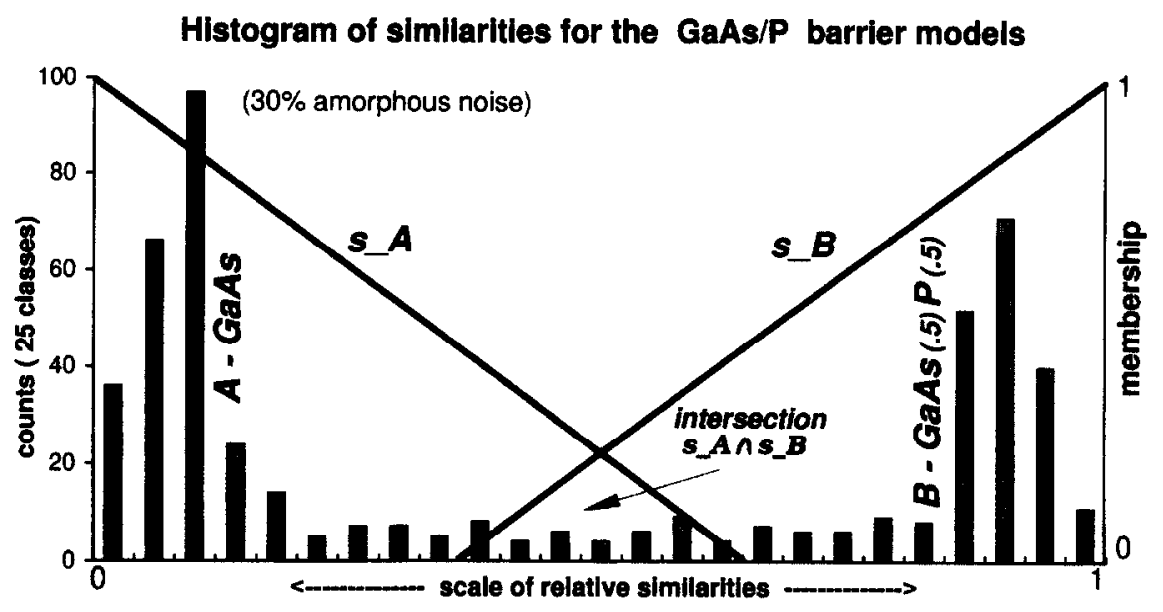

Fig. 5. Histogram of similarity values of the difference patterns $(16 \times 32$ cells of $32 \times 32$ pixels $)$; left: $G a A s(A)$, right: $G a A s_{0.5} P_{0.5}(B)$. Two examples of triangular FL membership functions $s_{-} A$ and $s_{-} B$ expressed by similarity values.

a transition range (barrier) in between. The shape and the height of the peaks are not the same for $A$ and $B$ because of their different share of the image area of Fig. 3 and the noise (see also Figs. 12 and 15).

\subsection{Basic concept of fuzzy logic}

Before the fuzzy logic set theory is applied to the HREM image analysis the FL methodology (sec, e.g. $[8,18,19])$ is introduced and described very briefly.

In fuzzy logic, information, characterized by a certain degree of truth, can be quantified and mathematically analysed. In 1965 , Zadeh [7] proposed a description of fuzzy information by graphs, expressing the degree of membership. The possibility of combining different membership functions then allows conclusions to be drawn on linguistic variables. In FL, membership functions are generally defined to express the degree of partially matching prototype properties in terms of set theory and logic. If the specific kind of the assumed membership functions fits the behaviour of the system, set theoretical operations allow quantitative conclusions to be drawn. The relations between the fuzzy sets are formulated by linguistic fuzzy rules of inference, as will be described in the next two sections. For studying the capacities of fuzzy logic in pattern recognition, dalabase systems, approximate reasoning and decision making as well as in engineering applications the reader is referred to [20].

Applying the FL formalism requires the variables of interest, i.e. similarity and composition to be expressed by the fuzzy sets in an adequate way for the whole range of definition. The characteristics of the membership functions (cf. figures below) such as the amount, support (base size), degree of intersection, and, cspccially, the shape (triangular, Gaussian, trapezoidal, ...) determine the reliability of the results obtained by applying the fuzzy rules formulated.

The values of similarity and composition derived are transformed into fuzzy logic membership functions. The quasi-linear behaviour compared to given prototypes of images allows triangular fuzzy sets to be defined by normalization and inversion, which will be demonstrated in the following sections where the fuzzy logic formalism is specified for the detection of compositional edges and for the chemical mapping of HREM micrographs of III-V compounds.

\subsection{FL approach to edge detection in HREM images}

The idea of the fuzzy logic algorithm derived in the following is to analyse similarity values of neighbouring image cells which are selected by 
means of appropriate masks. Generally, the size of the masks considered for identifying distinct image changes, called edges, depends on the nature and characteristics of the images (cf. [21]). Preliminary studies discussed in [22] showed that for HREM images of crystals $2 \times 3$ (or $3 \times 2$ ) masks are adequate because they keep a maximum number of interpretable image cells.

In Fig. 5 two fuzzy sets of similarity $s_{-} A$ and $s_{-} B$ are defined to classify the values in the histogram of similarity $S \in[0,1]$ of the GaAs/P image. In the region of intersection of both triangular membership functions the corresponding image cells are members of $A$ and $B$ simultaneously, i.e. they have medium composition. The two fuzzy sets are symmetric with the degree of intersection variable.

The image analysis is done by applying both fuzzy sets $S_{-} A$ and $s \_B$ to each of the six cells inside the $2 \times 3$ mask which gives the fuzzy variables $S_{i, j-1}, S_{i, j}, S_{i, j+1}, S_{i+1, j-1}, S_{i+1, j}$ and $S_{i+1, j+1}$. According to the fuzzy rules represented symbolically in Fig. 6 for edges parallel to the image borders (1) $(4)$, and for edges in diagonal direction $(5)-(8)$, fuzzy sets of composition are obtained. This way, transitions associated with both sides of an edge inside the mask are analysed.

The fuzzy variable of composition $C(i, j)$ represents the result of the inference. For the variable $C$ the two fuzzy sets $a$ (ltered) and $u$ (nchanged) are defined, where the set ' $a$ ' identifies the positions of edge-type similarity and composition, respectively. Fig. 6 contains solely those fuzzy rules which result in edge detection, i.e. they provide that $C(i, j)$ is $a$. By definition, for all other of the possible 32 cases $C(i, j)$ is $u$ is valid.

The fuzzy logic rules parallel to the borders of the image read:

(1) If $S_{i, j-1}$ is $A$ and $S_{i, j}$ is $A$ and $S_{i+1, j-1}$ is $B$ and $S_{i+1, j}$ is $B$, then $C(i, j)$ is $a$.

(2) If $S_{i, j-1}$ is $A$ and $S_{i, j}$ is $B$ and $S_{i+1, j-1}$ is $A$ and $S_{i+1, j}$ is $B$, then $C(i, j)$ is $a$.

(3) If $S_{i, j-1}$ is $B$ and $S_{i, j}$ is $A$ and $S_{i+1, j-1}$ is $B$ and $S_{i+1, j}$ is $A$, then $C(i, j)$ is $a$.

(4) If $S_{i, j-1}$ is $B$ and $S_{i, j}$ is $B$ and $S_{i+1, j-1}$ is $A$ and $S_{i+1, j}$ is $A$, then $C(i, j)$ is $a$.

Similarity values in $2 \times 3$ masks of image cells are compared to templates.

\begin{tabular}{l|l|l|l|}
\cline { 2 - 4 } $\begin{array}{l}\text { The values in the similarity } \\
\text { matrices are normallzed }\end{array}$ & $s i, j$ & $s i,+1$ \\
\hline & $s(+1, j-1$ & $s(+1, j$ & $s i+1, j+1$ \\
\hline
\end{tabular}

Two triangular fuzzy sets of similarity are defined: (s_A, s_B)

The analysed fuzzy sets of composition $C(i, j)$ read $(a, u)$ to $S(0,1)$.

(similiar to $A$ or $B$ )

(altered, unchanged)

\section{The following symbolized fuzzy rules identify edges}

$$
C(1, j)=a
$$

1. parallel to the borders
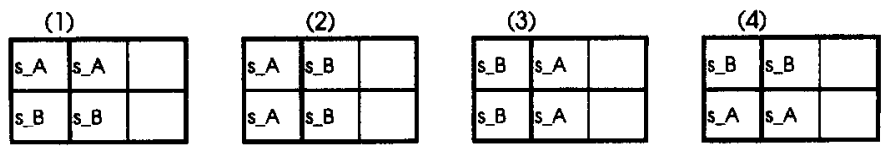

2. in diagunal direction
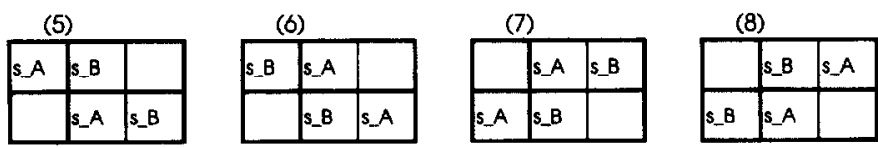

Fig. 6. Scheme of $2 \times 3$ matrices of neighbouring image cells, representing the fuzzy logic approach to 'edge detection'. 
For crystal type patterns the following diagonal rules are established:

(5) If $S_{i, j-1}$ is $A$ and $S_{i, j}$ is $B$ and $S_{i+1, j}$ is $A$ and $S_{i+1, j+1}$ is $B$, then $C(i, j)$ is $a$.

(6) If $S_{i, j-1}$ is $B$ and $S_{i, j}$ is $A$ and $S_{i+1, j}$ is $B$ and $S_{i+1, j+1}$ is $A$, then $C(i, j)$ is $a$.

(7) If $S_{i, j}$ is $A$ and $S_{i, j+1}$ is $B$ and $S_{i+1, j-1}$ is $A$ and $S_{i+1, j}$ is $B$, then $C(i, j)$ is $a$.

(8) If $S_{i, j}$ is $B$ and $S_{i, j+1}$ is $A$ and $S_{i+1, j-1}$ is $B$ and $S_{i+1, j}$ is $A$, then $C(i, j)$ is $a$.

Depending on the degree of intersection of the $A$ and $B$ fuzzy sets, the edge-type similarity features in the HREM patterns can be recognized more or less convincingly, according to the technique of defuzzification $[8,23]$. It has been checked that even for large variations of the degree of intersection of the triangular fuzzy sets (cf. Fig. 5) only one crystal cell in a monotonic gradient of composition is identified as an edge. An ambiguous repetition of bounds would suggest the misleading impression of composition oscillations. The formulated fuzzy rules exclude each other with respect to the conclusions. For this reason the defuzzification can be done by the fast "maximum method" [23], i.e. the highest degree of membership determines the result.

For demonstration the four-barrier model of Fig. 3 is analysed here. The FL rules are expected to identify and mark the trace of edge-like similarity changes in the image plane built up from $16 \times 32$ cells. Fig. 7 shows that parallel as well as diagonal oriented parts of the composition barriers fit the FL criteria, displayed by the value 1 for the corresponding cells. The positions of cells having minor compositional variations in the neighbourhood are depicted by zero-values. Because of the $2 \times 3$ mask size and the intentionally very rough four-barrier model "drop outs", as may be recognised by gaps in the interface, cannot be avoided.

\subsection{FL approach to chemical mapping of III-V compounds}

For the purpose of chemical mapping three fuzzy sets of similarity similar, medium and different are defined, abbreviated by $s, m, d$. They are to classify the values in the histograms of similarity gained by comparing the image cells to the templates $\mathrm{A}$ and $\mathrm{B}$,

\section{Fuzzy logic edge detection at GaAs/P}

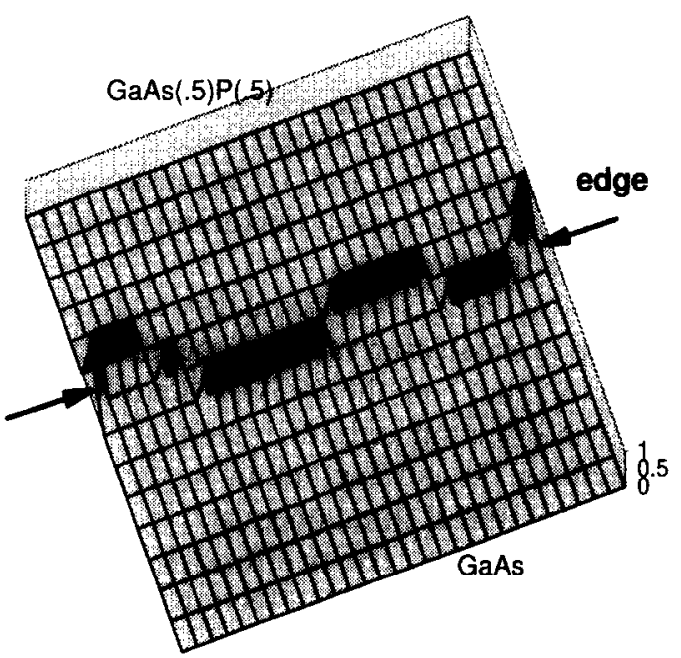

Fig. 7. Results of FL edge detection for the chemical barrier models, applying $2 \times 3$ masks. Note, comb $\equiv 1$ marks distinct changes in composition.

respectively. Both masks of neighbouring cells $(i, j)$ and $(i+1, j)$ have an identical position and their orientation is perpendicular to the interface, as it is shown in Fig. 8. The image analysis is done by a tailored formalism $[24,25]$ applying the fuzzy sets simultaneously to one $A$ - and one $B$-related mask which gives the fuzzy variables:

- $S_{i, j} A, S_{i+1, j}-A$ as related to $A-\mathrm{GaAs}$ and

- $S_{i, j-} B, S_{i+1, j-} B$ as related to $B-\mathrm{GaAs}_{0.5} \mathrm{P}_{0.5}$.

The fuzzy variable of composition $C(i, j)$ represents the result of the inference. For the variable $C$ the five fuzzy sets $\left[c_{-} A, c_{-} A m, c_{-} m, c_{-} m B, c_{-} B\right]$ are defined. Fig. 8 shows symbolically all useful combinations of fuzzy sets of similarity and the fuzzy rules that lead to the conclusions of chemical composition. The fuzzy rules for chemical mapping, numbered according to Fig. 8, read in mathematical notation:

(1) If $S_{i, j-} A$ is $s$ and $S_{i+1, j} A$ is $s$ and $S_{i, j-} B$ is $d$ and $S_{i+1, j} B$ is $d$, then $C(i, j)$ is $c_{-} A$.

(2) If $S_{i, j} A$ is $s$ and $S_{i+1, j} A$ is $m$ and $S_{i, j} B$ is $d$ and $S_{i+1, j-} B$ is $m$, then $C(i, j)$ is $c_{-} A m$.

(3) If $S_{i, j} A$ is $m$ and $S_{i+1 . j-A} A$ is $s$ and $S_{i, j} B$ is $m$ and $S_{i+1, j-} B$ is $d$, then $C(i, j)$ is $c_{-} A m$. 


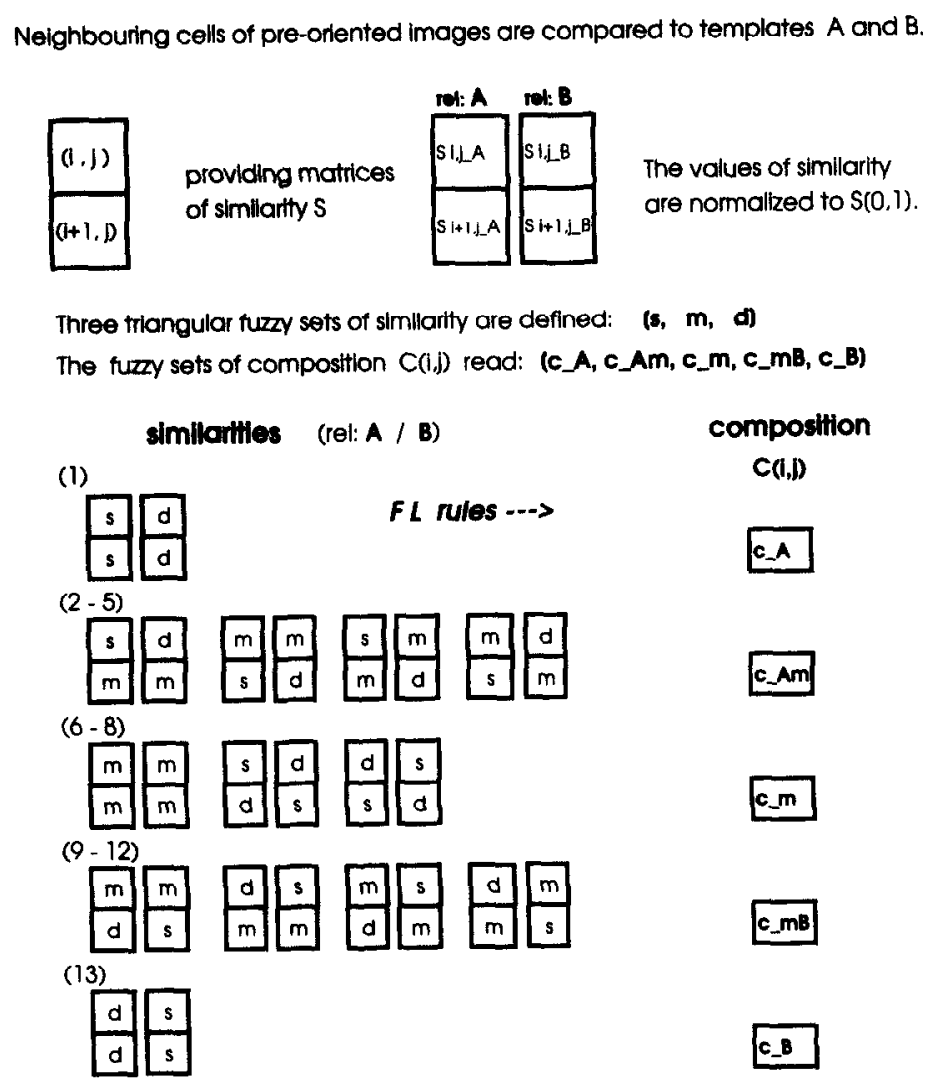

Fig. 8. Schemes of $2 \times 1$ matrices of neigbouring image cells (related to A and B) representing the fuzzy logic approach to "chemical mapping".

(4) If $S_{i, j-A}$ is $s$ and $S_{i+1, j-A} A$ is $m$ and $S_{i, j}-B$ is $m$ and $S_{i+1, j-} B$ is $d$, then $C(i, j)$ is $c_{-} A m$.

(5) If $S_{i, j-} A$ is $m$ and $S_{i+1, j-} A$ is $s$ and $S_{i, j} B$ is $d$ and $S_{i+1, j-B}$ is $m$, then $C(i, j)$ is $c_{-} A m$.

(6) If $S_{i, j-} A$ is $m$ and $S_{i+1, j-} A$ is $m$ and $S_{i, j-B} B$ is $m$ and $S_{i+1, j-} B$ is $m$, then $C(i, j)$ is $c_{-} m$.

(7) If $S_{i, j-} A$ is $S$ and $S_{i+1, j-} A$ is $d$ and $S_{i, j-B} B$ is $d$ and $S_{i+1, j} B$ is $s$, then $C(i, j)$ is $c . m$.

(8) If $S_{i, j}-A$ is $d$ and $S_{i+1, j}-A$ is $s$ and $S_{i, j-} B$ is $s$ and $S_{i+1, j-} B$ is $d$, then $C(i, j)$ is $c \_m$.

(9) If $S_{i, j} A$ is $m$ and $S_{i+1, j-} A$ is $d$ and $S_{i, j} B$ is $m$ and $S_{i+1, j-} B$ is $s$, then $C(i, j)$ is $c_{-} m B$.

(10) If $S_{i, j} A$ is $d$ and $S_{i+1, j} A$ is $m$ and $S_{i, j} B$ is $s$ and $S_{i+1, j-B} B$ is $m$, then $C(i, j)$ is $c \_m B$.

(11) If $S_{i, j} A$ is $m$ and $S_{i+1, j} A$ is $d$ and $S_{i, j-} B$ is $s$ and $S_{i+1, j-B}$ is $m$, then $C(i, j)$ is $c \_m B$.

(12) If $S_{i, j}-A$ is $d$ and $S_{i+1, j} A$ is $m$ and $S_{i, j-} B$ is $m$ and $S_{i+1, j-} B$ is $s$, then $C(i, j)$ is $c \_m B$.
(13) If $S_{i, j-} A$ is $d$ and $S_{i+1, j-} A$ is $d$ and $S_{i, j-} B$ is $s$ and $S_{i+1, j-} B$ is $s$, then $C(i, j)$ is $c_{-} B$.

If there are combinations of cell similarity values, which do not fulfil any of the FL rules (1)-(13), no composition can be concluded. Further fuzzy logic rules may be added to achieve some kind of "drop out" correction by allowing one of the four similarity conditions to be violated.

For histograms of the form given in Fig. 5 (see also Fig. 12) the fuzzy sets are specified by triangular membership functions. To illustrate the analysis in more detail, Fig. 9 shows the three triangular fuzzy sets of similarity $(s, m, d)$ used and the five fuzzy sets of composition obtained in a graphic representation. The measured values of similarity ( $x$-axis) related to A (left) and related to B (right) provide degrees of membership ( $y$-axis) for the 
Similarity and triangular fuzzy sets $\mathbf{s}, \boldsymbol{m}, \boldsymbol{d}$

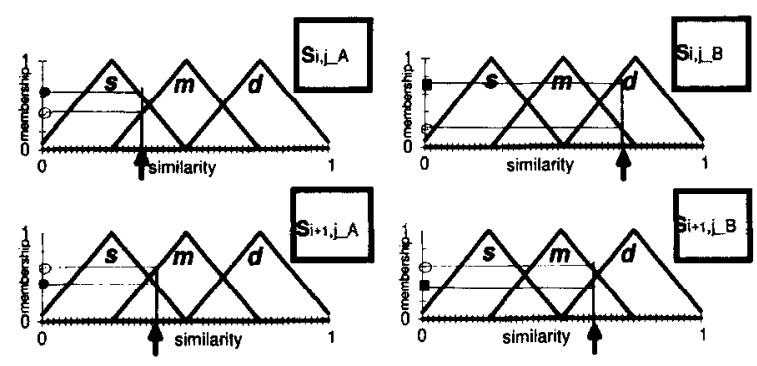

Fuzzy sets of composition $C \_A$... $C \_B$

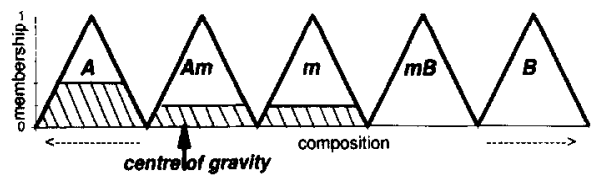

Fig. 9. Similarity values $S_{i, j}-A, S_{i+1, j}-A$ and $S_{i, j}-B, S_{i+1, j}-B$ of neighbouring cells registered in the triangular membership functions $s, m$ and $d$. FL rules "fill" the five fuzzy sets of composition. Defuzzification of the result is schematically represented at the bottom!

corresponding membership functions. The fuzzy rules (1)-(13) control the decisions on membership in the resulting sets of composition (bottom). The conjunction of logical ANDs in the fuzzy rules is given by the corresponding minimum of memberships (see $[18,19])$. The defuzzification illustratcd at the bottom of Fig. 9 is carried out by the centre of gravity method $[8,23]$, which has an interpolative effect. Physical prerequisites, such as the conservation of matter in the sub-lattices and the monotonicity of chemical gradients, are taken into account in this approach to HREM image analysis.

From this procedure of chemical mapping, the 3-D relief of composition shown for the GaAs/ $\mathrm{GaAs}_{0.5} \mathrm{P}_{0.5}$ barrier model in Fig. 10 is obtained. The fuzzy rules applied transform the variations of similarity values inside the $A$ - and $B$-related matrices to composition variations according to the quasi-linear characteristics of the triangular fuzzy sets. The amorphous noise is suppressed by the fuzzy classifications. The different slopes of the four barriers used in the contrast simulations can be clearly distinguished.

Aspects of applying the fuzzy logic approaches introduced in Sections 2.4 and 2.5 to experimental
Fuzzy logic composition mapping at GaAs/P

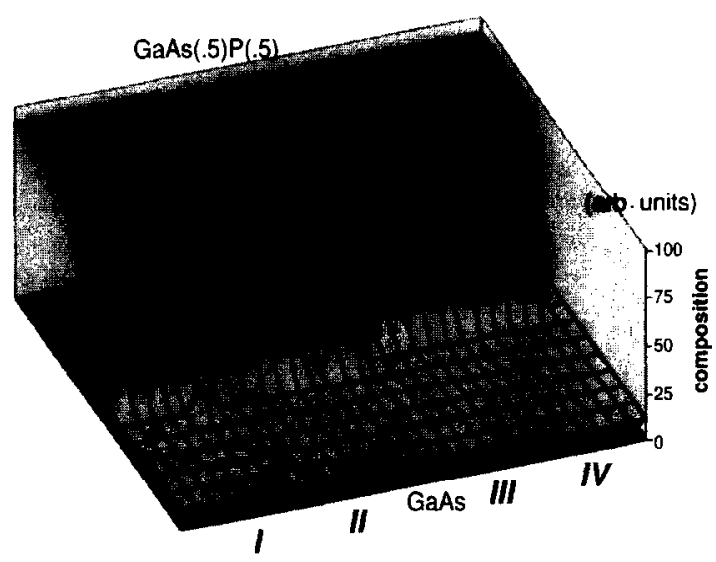

Fig. 10. Fuzzy logic chemical map of a simulated HREM image $(16 \times 32$ cells $)$ of four different $\mathrm{GaAs} / \mathrm{P}$ barriers with $30 \%$ amorphous noise.

HREM image analysis are discussed in the following section.

\section{Application to experimental HREM images}

The following applications refer to the established system (Al,Ga)As imaged with $\left\langle\begin{array}{llll}1 & 1 & 0\end{array}\right)$ crystal orientation in the electron microscope. For the $\left\langle\begin{array}{lll}1 & 1 & 0\end{array}\right\rangle$ crystal axis the electron diffraction of III-V sphalerite structures is non-centrosymmetric (polar) intensifying the non-linear relation between composition and similarity. This behaviour, e.g., studied in [17] by comparing the similarity profiles related to the two templates, is not critical with respect to edge detection. The FL analysis of varying composition gradients in 2.3 and 2.4 obviously provided unambiguous results of barrier detection. For chemical mapping, the deviations from linearity related to the different templates compensate each other partially as will be discussed later.

The first experimental example is a cross-section of an AlAs/ $/ \mathrm{Al}_{0.4} \mathrm{Ga}_{0.6}$ As interface in MBE-grown material. The HREM micrograph of Fig. 11 has image dimensions of about $11 \times 11 \mathrm{~nm}^{2}$. For studying the properties of the interface by edge detection as well as for extracting the $\mathrm{Al} / \mathrm{Ga}$-profiles, reference templates were generated from image areas 

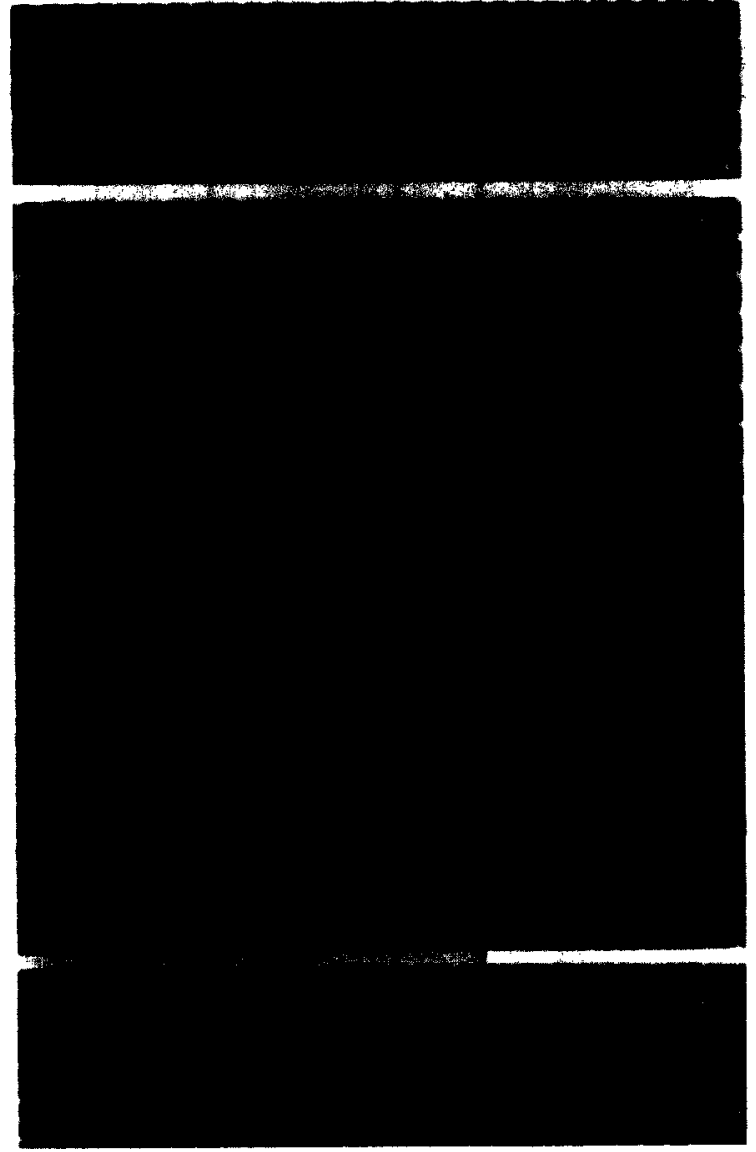

Fig. 11. HREM micrograph of an $\mathrm{AlAs} / \mathrm{Al}_{0.4} \mathrm{Ga}_{0.6} \mathrm{As}$ interface. $U=400 \mathrm{kV}, \mathrm{C}_{\mathrm{s}}=1 \mathrm{~mm}$, crystal orientation $\langle 110\rangle,(512 \times 512$ pixels, 8 bits); top and bottom: templates.

distant from the interface where the chemical composition is known from growth conditions and physical reference methods. Parts of the full-size patterns, averaged by Fourier filtering and/or accumulation techniques, which are used for image comparison are shown at the top and at the bottom of Fig. 11. The reference template $A$ (top) is related to AlAs and $\mathrm{B}$ (bottom) is related to $\mathrm{Al}_{0.4} \mathrm{Ga}_{0.6} \mathrm{As}$. The lateral mismatch between the generated templates and the crystal lattice in the HREM image, systematically studied in Section 2.1 , is reflected by the closest similarity and the slope attainable in the "S/cell shift-graph" (see Figs. 2 and 1) as well as by the histogram of similarity (cf. Fig. 14, Fig. 15).
The values of similarity may be presented as half-tone maps as it is shown in Fig. 12. Since it is not required that the cell size $(m \times n)$ should reproduce exactly the crystallographic unit cell for this kind of approach, $N$-row $=N \_$col $=32$ is chosen here. The darker a cell is displayed in the maps of Fig. 12 the larger is the dissimilarity, i.e. white represents closest similarity. At the border of the maps distortions arise, because the Fourier filtered template patterns do not fulfil the condition of periodic continuation. In addition to the similarity/composition relation, the type of template preparation, amorphous noise, lattice distortions and grid mismatch are reasons for deviations. A statistical analysis of the images provided an error bar of $7 \%$ with respect to the similarity values. In both maps which are nearly complementary the interface can be distinctly recognised.

The lower part of Fig. 12 shows the histograms of both maps of similarity. The graphs clearly show two well-distinguishable peaks with a range of similarity transitions (interface) in between. It is obvious that the form and the height of the peaks vary with the template used. The AlAs-template is of good symmetry with respect to both parts of the HREM image, whereas the AlGaAs template does not produce a narrow distribution of similarity values for the AlAs region of the micrograph.

The result of the complete FL image analysis of the $\mathrm{Al} / \mathrm{GaAs}$ micrograph (cf. Fig. 11) is summarized in Fig. 13. The upper part shows the output of the fuzzy logic edge detection procedure. The fuzzy rules (1-8) are in practice capable of providing a close trace of edge-type similarity/composition variation as visible in the image plane. Where no such edge-type variation is detected, cells are represented by zero-values. In this way the parameters of physical interest for III-V diffusion studies such as the position and course of the interface, can be clearly and easily derived. While the interface analysed here is relatively smooth, artificially roughened interfaces also can be interpreted successfully [22].

From the middle part of Fig. 13 the interpolating nature of applying fuzzy logic rules becomes clear. The chemical map of composition combines all information available from the two matrices of similarity values based on FL inference. 3D maps of 

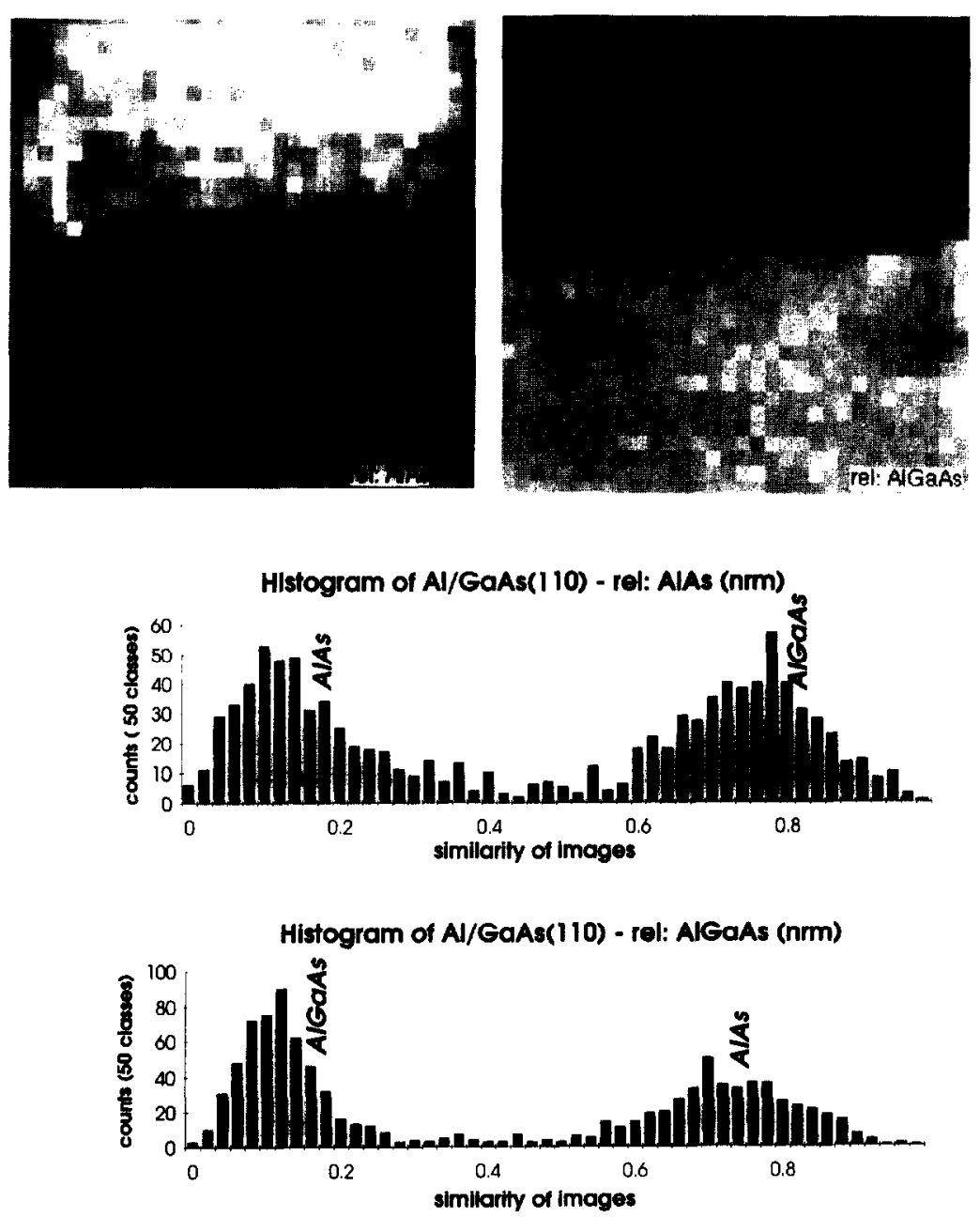

Fig. 12. Maps of cell similarities (top); left: related to AlAs; right: related to AlGaAs. Histograms of similarity values of the difference patterns (cells: $16 \times 16$ ), rel. AlAs and rel. AlGaAs (bottom).

composition provide interpretable and valuable profiles of the chemical diffusion gradient as shown in the bottom part of Fig. 13. For computing the profile, the two axes of the plot have to be calibrated: - the enlargement, the scale or the number of cells in the image ( $x$-axis),

- the chemical composition of the two templates (plateaus in the $y$-axis).

The parameters of physical interest for III-V diffusion studies such as the slope can clearly be derived.
In certain cases of multilayer systems the crystal lattice cannot be assumed to be uniform across the whole image area of HREM micrographs under investigation. This can be caused, for instance, by a lattice misfit between the two crystalline materials at the interface, by the growth conditions or other reasons. If there remains strain in the lattice it is nearly impossible to determine a grid that completely fits the matrix of image cells. As shown in Fig. 2 and checked in practice for the case given in Fig. 11, a grid-mismatch in the order of $25 \%$ of 


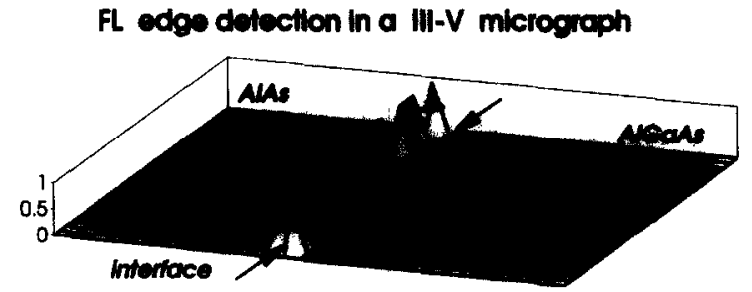

Fuzzy logic compoetilion map of N/CaAs

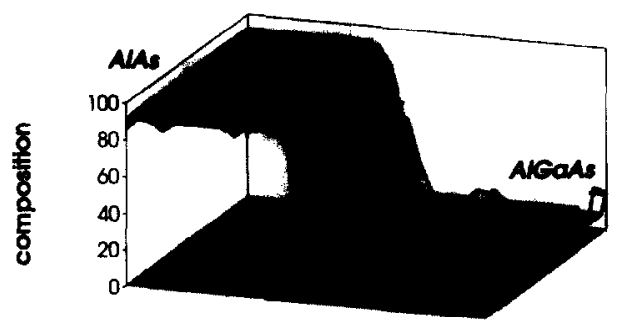

FL concentrotion profile of A/GaAs

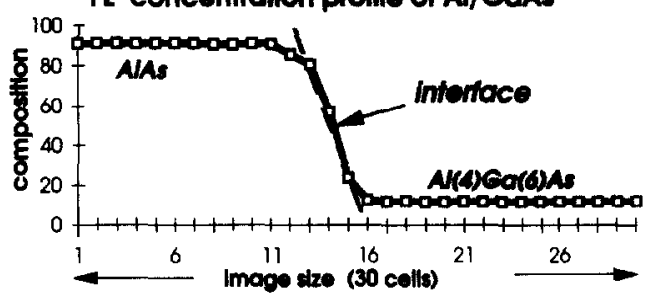

Fig. 13. Results of FL image analysis of the micrograph of Fig. 11. Edge detection (top), composition relief (middle) and concentration profile of $\mathrm{Al} / \mathrm{Ga}$ (bottom).

a cell size (asymmetric unit) disturbs the one-to-one correlation of similarity and composition.

Such conditions may be illustrated by the HREM micrograph of another interface in the system (Al,Ga)As shown in Fig. 14. It is obvious that the $\mathrm{Al} / \mathrm{Ga}$ composition, given from the growth conditions, seems to vary in the AlGaAs region. There are severe distortions of the periodicity in the lower part of the HREM image which are possibly intensified by thickness variations resulting from the preparation. It proved to be impossible to derive a representative template for the AlGaAs part. Three attempts to create templates of AlAs by Fourier filtering of various initial regions in the micrograph are shown by the respective difference patterns. A clear distinction of the two components adjacent to the boundary is impossible. The maps of cell similarity are affected by grid misfit and variations of the chemical composition of the same order of magnitude. To make the extracted cell similarities applicable to edge detection or chemical mapping in spite of these drawbacks the reference templates would have to be adapted to the crystal distortions cell by cell.

The corresponding histograms of the similarity maps shown in Fig. 15 provide profound hints on the selectivity of the templates applied. There is no case having a distinct minimum between the two peaks of similarity as it is expected for correct templates. The histograms of templates 1 and 3 reveal something like a minimum, but, as Fig. 14 clearly shows, the two weak maxima can not be related to the III-V composition at both sides of the interface. Further, HREM micrographs of the material system $\mathrm{Al} / \mathrm{GaAs}$ provided similarity maps of different quality. Whereas the edge detection algorithm clearly could identify an interface even if only one template was of sufficient quality, the FI. chemical mapping technique required both matrices of cell similarity to be suitable.

The last experimental example has been chosen to point out the limitations of the approach to HREM of III-V compounds designed. It is not a limitation of the FL technique of image analysis but a problem of data acquisition in pre-processing. In all cases the maps of cell similarity as well as the histograms allow to determine the reliability of image analysis. Improving the sensitivity of the approach to experimental applications requires the individual fit of the templates cell by cell to compensate geometrical imperfections of the crystalline images.

\section{Conclusions}

A novel fuzzy logic approach to the HREM image analysis has been developed and used for structural and chemical analyses of III-V semiconductor interfaces. The analysis of HREM patterns is based on the determination of similarities of image details in relation to templates (basic image patterns) of known crystal composition. The values of similarity are transformed into adapted fuzzy 


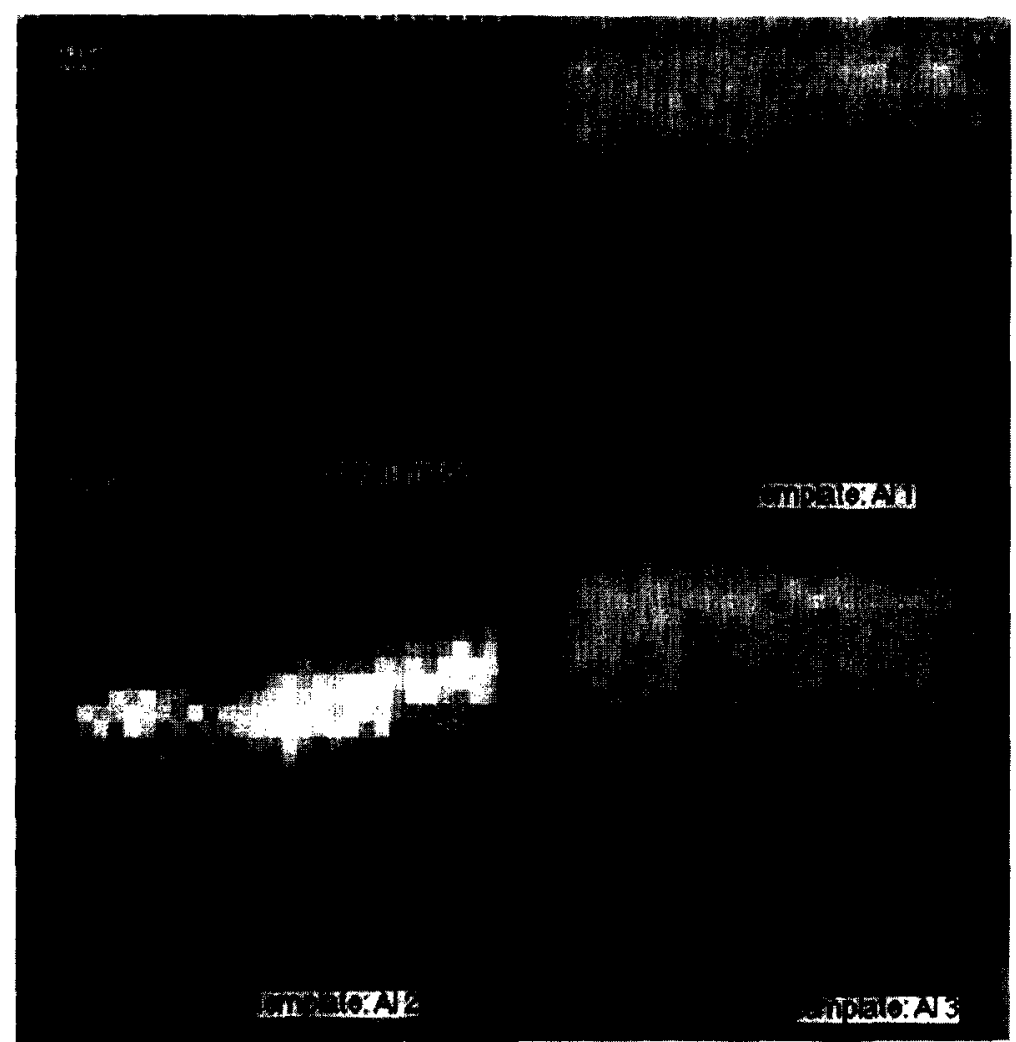

Fig. 14. HREM micrograph of a $\langle 1 \mid 10\rangle$ oriented crystal with AlAs $/ \mathrm{AlGaAs}$ interface (a). $U=400 \mathrm{kV}, \mathrm{C}_{\mathrm{s}}=1 \mathrm{~mm}(512 \times 512$ pixels, 8 bits) and maps of cell similarity for three different AlAs-templates (b)-(d).

logic membership functions to profit from the abilities of $\mathrm{FL}$ in image processing.

A formalism of edge detection is introduced to distinguish crystal regions with different properties. These edges (interfaces, barriers) are defined by relatively distinct changes of the image patterns. The fuzzy rules derived for neighbouring image cells $(2 \times 3$ masks $)$ combine two triangular membership functions of similarity to give conclusions of distinct alterations of composition.

The paper presents examples of the successful application of this algorithm to the localization of edge-type features in HREM images. The reliability of the fuzzy rules derived is demonstrated for various interface models by evaluating simulated contrast tableaus of $\mathrm{GaAs} / \mathrm{GaAsP}\left\langle\begin{array}{lll}0 & 0 & 1\end{array}\right\rangle$ interface regions. The FL edge detection applied to experi- mental micrographs of the system (Al,Ga)As $\left\langle\begin{array}{lll}1 & 1 & 0\end{array}\right\rangle$ enables the position of the III-V interface region to be detected.

The fuzzy logic rules formulated for the determination of the chemical composition allow us to analyse the similarity gradient of neighbouring image cells $(2 \times 1$ mask $)$ in relation to two templates of different, but known composition. To this purpose, the values of similarity are transformed into triangular fuzzy logic membership functions to draw inference from the $\mathrm{FL}$ rules locally fulfilled.

The FL approach of composition analysis was applied to simulated HREM images of GaAs/ $\mathrm{GaAsP}\langle 001\rangle$ interface models. In its reliability the FL analysis compares with the "chemical mapping" technique $[1,12]$ developed by Ourmazd et al. A critical analysis of the results obtained 


\section{Hiatcgrams of AVGaAs(110) for different AlAs templates}
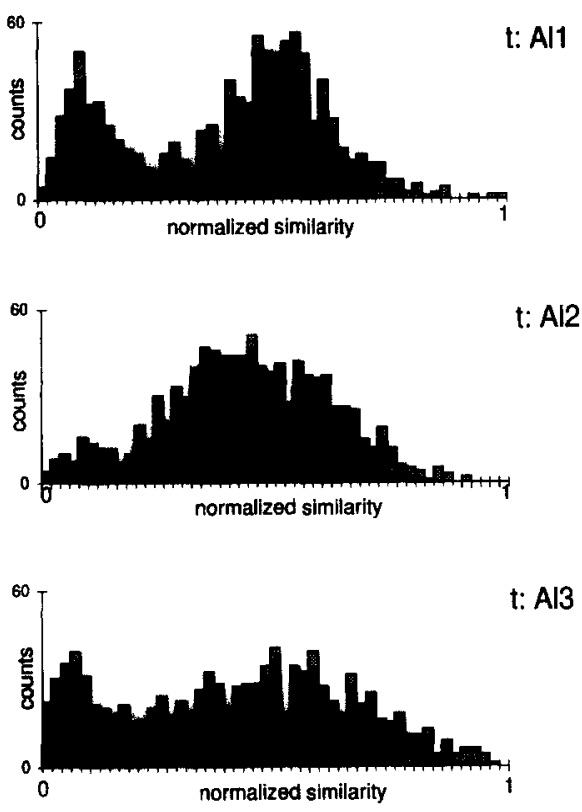

Fig. 15. Histograms of similarity values of the difference patterns (cells: $16 \times 16$ ) for three different AlAs templates.

showed that the FL approach provides reasonable results even under non-linear conditions of $\langle 110\rangle$ crystal orientation. The deviation of the determined composition from the true one can be estimated by comparing the similarity characteristics of both templates with respect to their complement. The evaluation of experimental HREM material of III-V crystals proved to be successful in providing valuable results.

The lateral resolution of both methods is of the order of one projected unit cell. The respective masks are about $1 \mathrm{~nm} \times 1 \mathrm{~nm}$ in size. The exact course of barriers as well as the composition gradient related can be quantified by fuzzy logic conclusions. It is noteworthy that the proposed FL approach is implemented on PC platform and can easily be adapted to commercial image processing software. By the definition of adapted, new sets of fuzzy rules the approach can easily be extended to the analysis of other image features.

\section{Acknowledgements}

The authors are grateful to P. P. Wang (Durham, NC) for suggestions in theoretical image processing and fuzzy logic, and to Ch. Bauer (Halle, Germany) for stimulating discussions. The authors would like to thank R. Hey, Paul Drude Institute Berlin, for the MBE-grown samples of AlGaAs heterostructures.

\section{References}

[1] A. Ourmazd, F.H. Baumann, M. Bode and Y. Kim, Ultramicroscopy 34 (1990) 237.

[2] P. Schwander, C. Kisielowski, M. Seibt and F.H. Baumann, Y. Kim and A. Ourmazd, Phys. Rev. Lett. 71 (1993) 4150.

[3] A.F. De Jong and D. Van Dyck, Ultramicroscopy 33 (1990) 269.

[4] S. Thoma and H. Cerva, Ultramicroscopy 35 (1991) 77.

[5] T. Walther, D. Gerthsen, R. Carius, A. Förster and K. Urban, in: Microscopy of Semiconducting Materials, Oxford, 1993, Inst. Phys. Conf. Ser. Vol. 134, (1993) p. 449.

[6] C.-Y. Tyan and P.P. Wang, Proc. 2nd IEEE Conf. on Fuzzy Systems, San Francisco (1993) p. 660.

[7] L.A. Zadeh, Fuzzy sets, Inform. and Control 8 (1965) 338.

[8] H.-J. Zimmermann, Fuzzy Set Theory - and Its Applications 2nd rev. ed. (Kluwer Academic Publishers, Dordrecht, 1991).

[9] T.Y. Tan, U. Gösele and S. Yu, Crit. Rev. Solid State Mater. Sci. 17 (1991) 47.

[10] D. Stenkamp and W. Jäger, Ultramicroscopy 50 (1993) 321.

[11] P. Stadelmann, Ultramicroscopy 2 (1987) 131.

[12] A. Ourmazd, Mater. Sci. Rep. 9 (1993) 201.

[13] R. Hillebrand and H. Hofmeister, Phys. Stat. Sol. (a) 150 (1995) 65.

[14] E. Diday and J.C. Simon, Digital Pattern Recognition, Ed. K.S. Fu (Springer, Berlin, 1976) p. 51.

[15] R. Hillebrand, H. Hofmeister, P. Werner and U. Gösele, Appl. Phys. Lett. 67 (1995) 1763.

[16] C. Kisielowski, P. Schwander, F.H. Baumann, M. Seibt, Y. Kim and A. Ourmazd, Ultramicroscopy 58 (1995) 131.

[17] R. Hillebrand, P. Werner, H. Hofmeister and U. Gösele, in: Microscopy of Semiconducting Materials, Oxford, 1995, Inst. Phys. Conf. Ser. Vol. 146, (1995) p. 57.

[18] M. Seraphin, Neuronale Netze und Fuzzy-Logik (Franzis', München, 1994).

[19] W. Pedrycz, Fuzzy Control and Fuzzy Systems 2nd extended ed. (Wiley, New York, 1993).

[20] G.J. Klir and B. Yuan, Fuzzy Sets and Fuzzy Logic: Theory and Applications (Prentice-Hall, Englewood Cliffs, NJ, 1995). 
[21] C.Y. Wang, Edge Detection Using Template Matching, $\mathrm{Ph}$. D. Dissertation, Duke University (Michigan Dissertation Microfilm) (1985).

[22] R. Hillebrand, P. Wang and U. Gösele, Inform. Sci. Appl. 93 (1996) 321.

[23] J. Kahlert and H. Frank, Fuzzy-Logik und Fuzzy-Control (Vieweg, Braunschweig, 1993)
[24] R. Hillebrand and U. Gösele, in: Proc. 2nd Joint Conf. on Information Sciences Wrightsville Beach, NC (1995) p. 378.

[25] R. Hillebrand and U. Gösele, Inform. Sci. Appl., in press. 